\title{
Consider Nitrofurantoin as a Cause of Lung Injury
}

\author{
Paulo Almeida ${ }^{1}$, Eduarda Seixas ${ }^{2}$, Beatriz Pinheiro ${ }^{1}$, Pedro Ferreira $^{2}$, Ana Araújo ${ }^{1}$ \\ ${ }^{1}$ Department of Internal Medicine Centro Hospitalar Baixo Vouga, Aveiro, Portugal \\ ${ }^{2}$ Department of Pneumology, Centro Hospitalar Baixo Vouga, Aveiro, Portugal
}

Received: 24/09/2019

Accepted: $15 / 10 / 2019$

Published: 30/10/2019

How to cite this article: Almeida P, Seixas E, Pinheiro B, Ferreira P, Araújo A. Consider nitrofurantoin as a cause of lung injury. EJCRIM 2019;6: doi:10.12890/2019 001295.

Conflicts of Interests: The Authors declare that there are no competing interest

This article is licensed under a Commons Attribution Non-Commercial 4.0 License

\section{ABSTRACT}

Nitrofurantoin-induced diffuse lung toxicity is well documented in the literature but is often misdiagnosed. We describe an 82 -year-old female medicated with nitrofurantoin for the previous 2 years who was admitted for dyspnoea, dry cough and fatigue for 4 months. She was febrile and tachypnoeic and she presented with bilateral basal crackles, hypoxaemic respiratory failure and slightly elevated C-reactive protein levels. A chest radiograph showed bilateral air-space consolidation and interstitial infiltrates and the high-resolution computed tomography scan was evocative of a perilobular pattern of organising pneumonia (OP). Due to the clinical-radiological context, she was diagnosed with a presumable nitrofurantoin-induced OP. She was started on prednisolone $60 \mathrm{mg}$ daily with a progressive improvement. It is important that clinicians are aware of the spectrum of side effects associated with nitrofurantoin so as to monitor patients.

\section{LEARNING POINTS}

- It is crucial to ensure that a thorough medical history with a systems review and a complete drug history are carried out.

- Chronic pulmonary toxicity due to nitrofurantoin is rare and it occurs primarily in older women who have been prescribed relatively small doses of nitrofurantoin for UTI prevention.

- The cessation of nitrofurantoin is the basis of the treatment and may be sufficient for clinical and radiological improvement.

\section{KEYWORDS}

Nitrofurantoin, pulmonary toxicity, drug-induced lung toxicity, organising pneumonia

\section{CASE DESCRIPTION}

An 82-year-old Caucasian female was admitted to the medical ward with worsening dyspnoea, shortness of breath, longstanding dry cough, intermittent fever and intense fatigue for approximately 4 months. She was hospitalised approximately 3 months previously due to a community-acquired pneumonia and treated with empiric antibiotherapy with a temporary improvement. She had hypertension controlled with losartan and urinary incontinence medicated with trospium chloride. No other medication was initially reported. She was a non-smoker with no occupational exposure or allergies. Upon examination, she was febrile $\left(38.0^{\circ} \mathrm{C}\right)$, tachypnoeic and hypoxaemic with an $\mathrm{SpO} 2$ of $87 \%$ on room air. Chest auscultation revealed bilateral basal inspiratory crackles. Laboratory workup showed elevated C-reactive protein (3.0 $\mathrm{mg} / \mathrm{dl}[<0.50])$ and lactate dehydrogenase (1498 U/I [120-246]) levels and hypoxaemic respiratory failure (PaO2/FiO2 ratio=235). A chest radiograph (CXR) exposed bilateral air-space consolidation and interstitial infiltrates (Fig. 1A).

During the hospital stay, a slight hypertransaminasaemia (AST $147 \mathrm{U} / \mathrm{I}[<40]$ and ALT $142 \mathrm{U} / \mathrm{l}$ [<49]) was noted with a positive antimitochondrial M2 antibody. Blood cultures and viral serologies were negative. Thyroid function, brain natriuretic peptide, abdominal ultrasonography and echocardiogram assessments were normal. A high-resolution computed tomography scan (HRCT) revealed large, 
bilateral and basal areas of patchy alveolar infiltration, bilateral ground-glass opacification and some thick-walled bronchi with distortion (Fig. 2). The pattern was highly suggestive of a "perilobular variant" of organising pneumonia (OP). Flexible bronchoscopy was unremarkable and the bronchoalveolar lavage (BAL) presented a cellular profile compatible with OP (63\% lymphocytes [CD4/CD8 ratio 0.7], 8\% neutrophils and $26 \%$ macrophages). Cytological, bacteriological and mycobacteriological examinations of BAL were negative. Lung function testing revealed a restrictive pattern [Tiffeneau index 0.76, FVC 62.4\% predicted] with a diffusing capacity of the lungs for carbon monoxide (DLCO) of $29.7 \%$ predicted. A thorough medical history with a systems review was conducted and the patient reported recurrent urinary tract infections (UTI) that resolved after taking a "vitamin" advised by a family member. Upon accessing the computer drug-dispensing software, it was discovered that the patient was taking nitrofurantoin 100 mg every day for the past 2 years that had been prescribed by the family doctor at her request.

Within the described context, a diagnosis of nitrofurantoin-induced OP was assumed. Due to old age and the presence of an elusive perilobular pattern for OP, a decision was made not to pursue biopsy. The patient was started on prednisolone $0.75 \mathrm{mg} / \mathrm{kg} / \mathrm{day}$ with a rapid and sustained improvement making her discharge possible. The drug was obviously discontinued. After 12 weeks the patient was asymptomatic with a normal laboratory workup, a 20\% improvement in FVC (82\% predicted), a 16\% improvement in DLCO (45.6\% predicted) and radiographic normalisation (Fig. 1B). She completed the planned tapering of prednisolone with clinical stability.

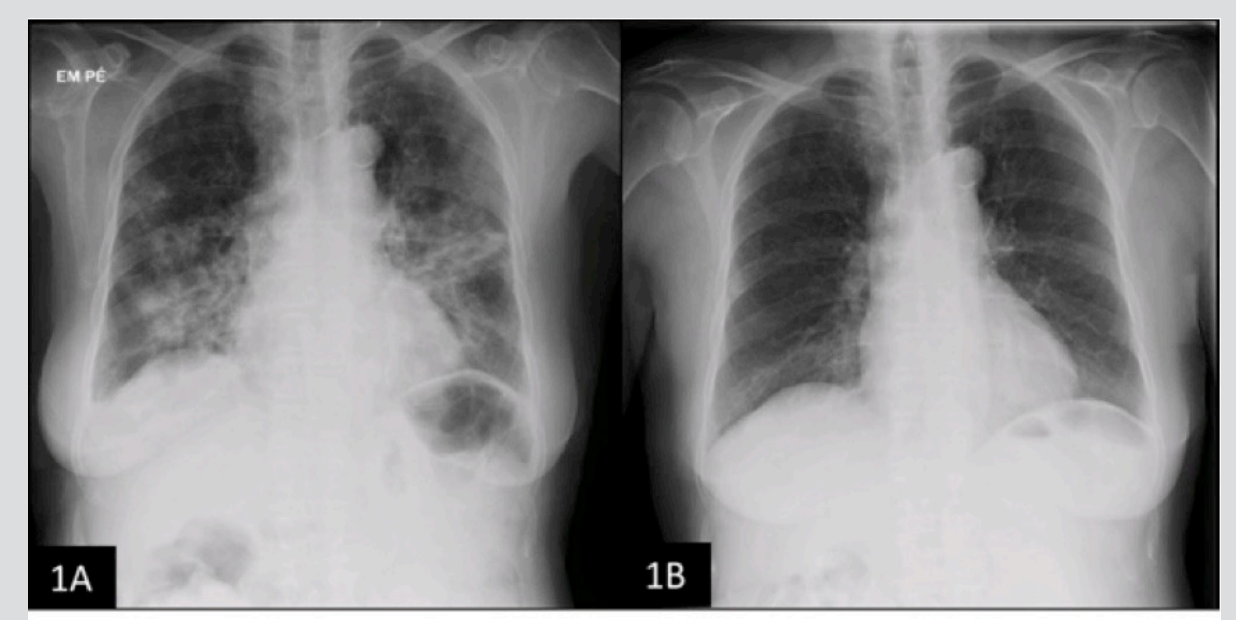

Figure 1. 1A: Chest radiograph (CXR) at patient's admission with bilateral air-space consolidation and interstitial infiltrates. 1B: Follow-up CXR at 12 weeks showing resolution of the previous parenchymal abnormalities

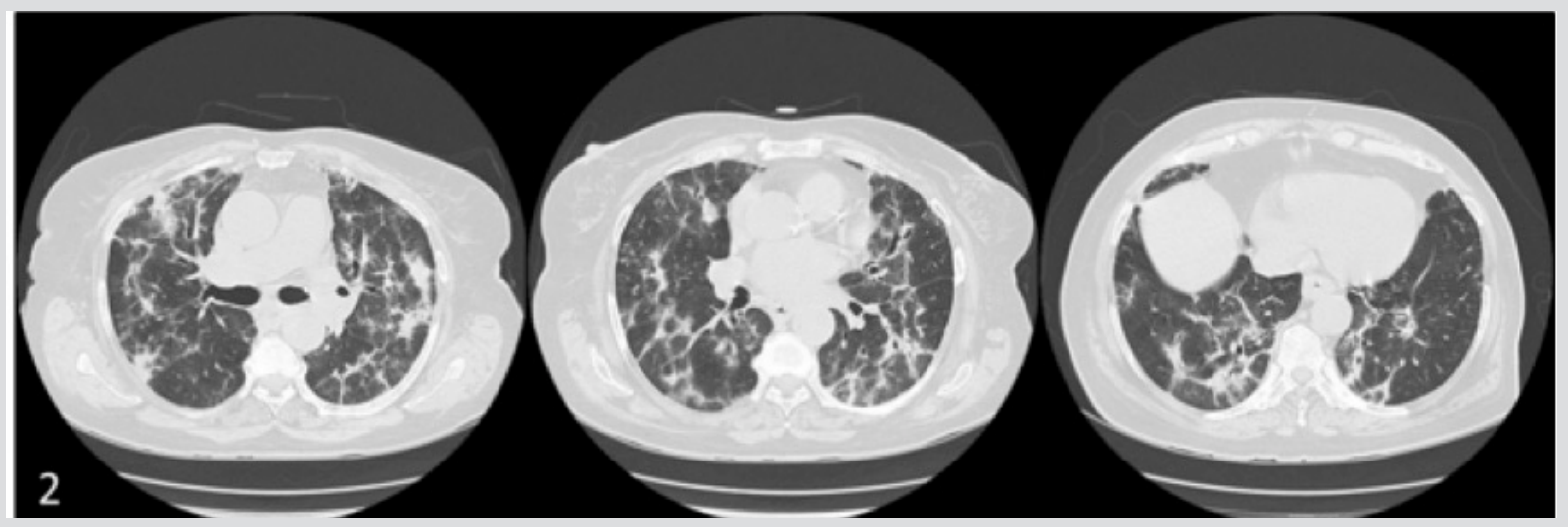

Figure 2. The high-resolution computed tomography scan (HRCT) revealed large and bilateral areas of patchy alveolar infiltration and linear opacities, the largest with evident are as of an air bronchogram, mainly at the bases; bilateral ground-glass opacification, involving the most organised densification areas; some thick-walled bronchi with distortion and appereance suggestive of small traction bronchiectactis 


\section{DISCUSSION}

Nitrofurantoin is an antibacterial agent commonly used for treatment and prevention of UTI. The incidence of pulmonary toxicity due to nitrofurantoin is estimated to lie between $0.0001 \%$ and $0.001 \%{ }^{[1]}$. Although rare, nitrofurantoin-induced diffuse lung toxicity is one of the most commonly reported pulmonary drug toxicities, reflecting the increased popularity of the drug ${ }^{[2]}$. Female sex, accountable for $85-95 \%$ of cases, renal impairment and older age are associated with higher risk of this toxicity ${ }^{[3,4]}$. This phenomenon is not dose-related and can have an acute onset (approximately $90 \%$ of cases) or a chronic presentation ${ }^{[1,3]}$. Chronic pulmonary toxicity is seen primarily in older women who have been prescribed relatively small doses of nitrofurantoin for UTI prevention ${ }^{[3,4]}$. Clinical presentation is nonspecific and less intense than in acute reactions, making diagnosis more challenging. There is a delay between the time of initial exposure to nitrofurantoin and the insidious onset of symptoms. The initial complementary examinations are focused on excluding other diagnoses, notably other interstitial lung diseases. Laboratory abnormalities may include positive anti-nuclear, anti-smooth muscle and anti-neutrophil cytoplasmic antibody results ${ }^{[3,4]}$, as in our patient, and may not indicate a rheumatic disease. Bilateral and basal lung infiltrates are seen on CXR in $94 \%$ of patients ${ }^{[1]}$. The HRCT shows frequently bilateral ground-glass attenuation, irregular linear opacities, bibasal air-space consolidation and fibrosis [1,2.5]. $^{2}$ Lung function assessment generally reveals restrictive physiology and the BAL cellular profile can reveal lymphocytosis, neutrophilia or eosinophilia ${ }^{[3]}$. Lung biopsy is not always necessary in a highly suspicious context ${ }^{[3]}$.

The cessation of nitrofurantoin is the basis of the treatment and may be sufficient for clinical and radiological improvement ${ }^{[2,3]}$. Re-exposure to nitrofurantoin is not advised due to the high likelihood of recurrence ${ }^{[3,5]}$. For patients with severe respiratory compromise, as for our patient, glucocorticoids can be necessary and a prednisolone-equivalent dose of $0.75 \mathrm{mg} / \mathrm{kg}$ per day is initially given for the first 1 to 3 months, with a tapering regimen as tolerated for a total duration of 6-12 months ${ }^{[3,5]}$. Most patients will improve after 2 to 12 weeks, although mild fibrotic changes persisted long-term in two-thirds of patients ${ }^{[3]}$.

This reaction is well documented in the literature, but is often misdiagnosed, resulting in unnecessary treatment, longer hospital stay and increased morbi-mortality ${ }^{[2]}$. Similar to other cases, our patient was initially admitted for presumed pneumonia. The score of 6 on the Naranjo Adverse Drug Reaction Probability Scale indicated that the patient's OP was probably related to nitrofurantoin. Thus, a definitive diagnosis of a secondary OP pattern of lung injury related to nitrofurantoin was made in light of: a compatible history of exposure to nitrofurantoin; restrictive lung physiology; a HRCT pattern of OP; a supporting BAL profile; exclusion of other diagnoses; a clear clinical and radiological improvement after cessation of the drug and corticosteroid therapy.

In the case described, the patient did not recognise nitrofurantoin as a drug with toxic potential and therefore did not report taking it. On the other hand, the medical team tends to communicate poorly with patients, relying too much on computer records to make the medical history. Thorough medical history and therapeutic reconciliation must be part of every physician's routine. In addition, to avoid similar cases, communication between physicians, carers and pharmacists should be improved and the population should be educated regarding the iatrogenic potential of any drug. When clinicians cannot avoid the use of nitrofurantoin, they should be aware of the potential lung injury and carefully monitor patients.

\section{REFERENCES}

1. Browning ET, Huckleberry JM, Barrow WB, Restauri NL, Kemme DJ, Cool CD, et al. Downstaging of non-small-cell lung cancer through identification of reversible drug toxicity. J Clin Oncol 2016;34:e6-8.

2. Kabbara WK, Kordahi MC. Nitrofurantoin-induced pulmonary toxicity: a case report and review of the literature. J Infect Public Health 2015; $309-313$.

3. de Zeeuw J, Gillissen AG. Nitrofurantoin-induced pulmonary injury. UpToDate 2018; http://www.uptodate.com (accessed September 1, 2019)

4. Roden AC, Camus P. latrogenic pulmonary lesions. Semin Diagn Pathol 2018;35:260-271.

5. Kanji Z, Su VC, Mainra R. Nitrofurantoin-induced pulmonary reaction involving respiratory symptoms: case report. Can J Hosp Pharm 2011;64:362-365. 